UDK 535:530.182

\author{
*Davletov A.E., Kudyshev Zh.A., Mukhametkarimov Ye.S., Kissan A., Yerimbetova L.T. \\ Department of Plasma Physics, al-Farabi Kazakh National University, \\ Tole Bi 96, Almaty 050012, Kazakhstan \\ *e-mail:askar@physics.kz
}

\title{
Dispersion of electromagnetic waves in layered graphene-dielectric metamaterials
}

\begin{abstract}
In this paper possibility of creating a medium based on graphene-dielectric (quartz) structures with a hyperbolic shape of the dispersion relation is considered. Using the Bloch theorem and the transfer matrix method, dispersion relations are found for hyperbolic media, consisting of periodically located layers of graphene and a dielectric. The transfer matrix method gives the possibility to relate the amplitudes of the incident and reflected electromagnetic waves at the input to the layered structure with the amplitudes at the output by means of propagation and transfer matrixes. The frequency dependent optical conductivity of graphene sheet is calculated by Kubo formula which takes into account both interband and intraband transitions. It is shown that when the chemical potential of graphene is increased, the elliptic form of the dispersion curve turns into to hyperbolic. This result is due to the fact that the perpendicular component of the permittivity changes its sign when the chemical potential is changed.
\end{abstract}

Key words: hyperbolic metamaterials, graphene, dispersion of electromagnetic waves.

\section{Introduction}

Strongly anisotropic media, in which the components of the diagonal tensor of permittivity or permeability have opposite signs, are called hyperbolic media or hyperbolic metamaterials (HMM) [1]. These materials are of great practical interest associated not only with the comparative ease of their fabrication, but mostly with their unusual optical properties. It is known that the hyperbolic metamaterials, along with the negative refraction of light [2], can be used to overcome the diffraction limit [3-6], to realize optical resonators and waveguides at the nanoscale $[7,8]$, to develop biosensor applications [9] and to enhance nonlinear effects [10]. In most cases, the hyperbolic metamaterials are obtained on the basis of a composition of metal nanoparticles immersed into dielectric media. There are two main methods for obtaining materials with the hyperbolic dispersion. In the first case, the hyperbolic response is achieved due to alternating layers of metal and dielectric, and the second is due to metal nanowires, arranged in a dielectric medium.

At present, research is actively under way on the development of the hyperbolic materials based on a graphene. The graphene, which is a single layer of graphite, has very unique electrical, magnetic and thermal properties [11-13]. Electrons in the graphene behave like the photons in a vacuum, i.e. they have a zero effective mass, which leads to a linear dispersion law [14]. In addition, strong plasmon effects are observed in the graphene, which, if necessary, can be influenced by doping [12].

In this paper possibility of creating a medium based on graphene-dielectric (quartz) structures with a hyperbolic shape of the dispersion relation is considered (Figure 1).

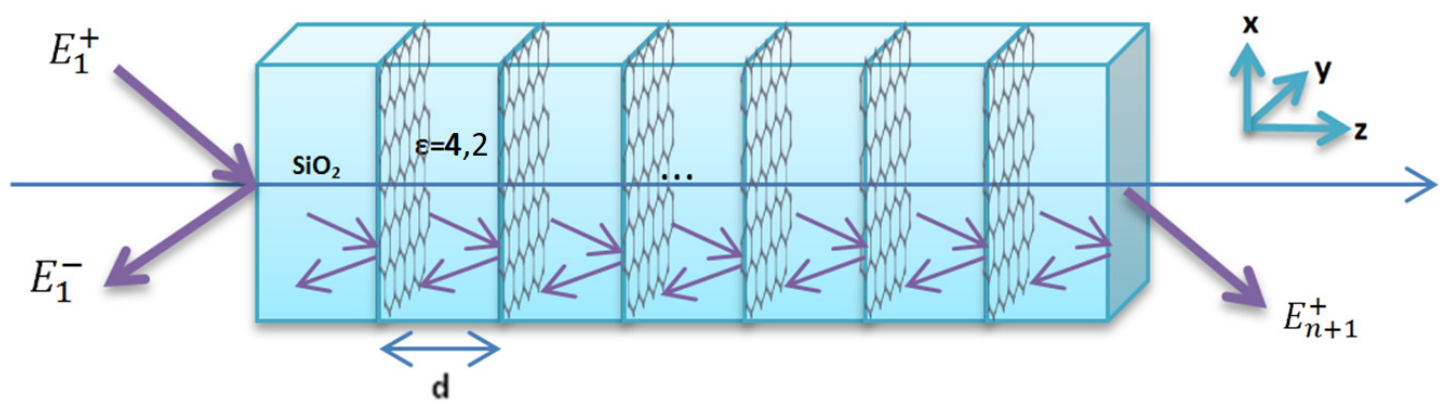

Figure 1 - Layout of layers of periodic structure based on dielectric and graphene 


\section{Optical conductivity of graphene}

The electromagnetic properties of grapheme sheets are characterized by the surface conductivity $\sigma$ within the Kubo formalism. In the absence of an external magnetic field, the surface conductivity of grapheme sheet is defined as a sum of the intraband and interband conductivities as:

$$
\sigma=\sigma_{\text {intra }}+\sigma_{\text {inter }}
$$

where

$$
\begin{gathered}
\sigma_{\text {intra }}=\frac{\mathrm{ie}^{2} \mathrm{k}_{\mathrm{b}} \mathrm{T}}{\pi \hbar^{2}(\omega+\mathrm{i} 2 \gamma)}\left(\frac{\mu}{\mathrm{k}_{\mathrm{b}} \mathrm{T}}+2 \log \left[\mathrm{e}^{-\frac{\mu}{\mathrm{k}_{\mathrm{b}} \mathrm{T}}}+1\right]\right), \\
\sigma_{\text {inter }}=\frac{\mathrm{ie}^{2}}{4 \pi \hbar^{2}} \log \left[\frac{2 \mu-(\omega+\mathrm{i} 2 \gamma) \hbar}{2 \mu+(\omega+\mathrm{i} 2 \gamma) \hbar}\right] .
\end{gathered}
$$

Here $\hbar$ denotes the Plank constant, $\mu$ is the chemical potential, $\gamma$ refers to the electron-phonon scattering rate, $\omega$ designates the incident wave frequency, $\mathrm{k}_{\mathrm{b}}$ stands for the Boltzmann constant, $\mathrm{T}$ signifies the temperature and $\mathrm{e}$ is simply the elementary charge.

Figure 2 displays the imaginary and real parts of the surface conductivity, which is normalized to $\sigma_{0}=\frac{e^{2}}{4 \hbar}$.

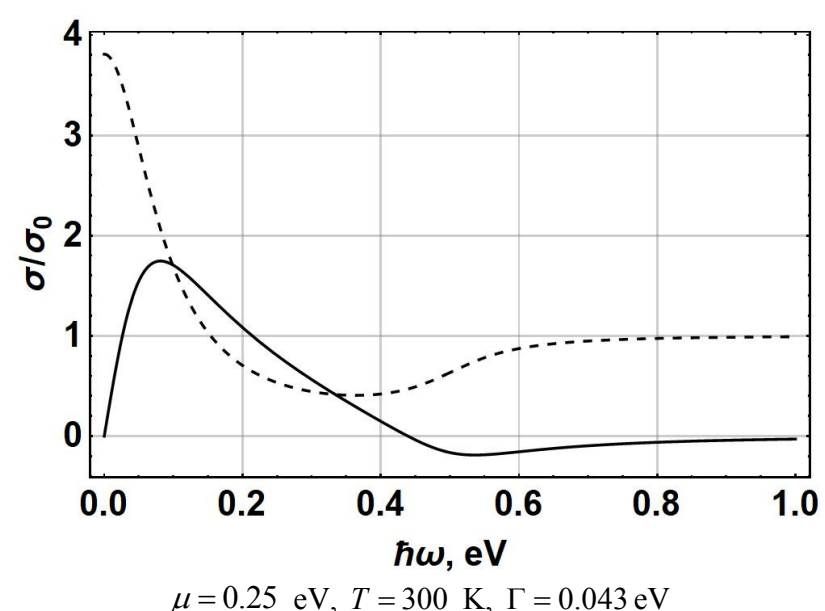

Figure 2 - Optical conductivity of the graphene sheet. Solid line: the imaginary part; dashed line: the real part.

The matrix method or the matrix transfer method makes it possible to relate the amplitudes of the incident and reflected electromagnetic waves at the input to the layered structure with the amplitudes at the output by means of the matrix $M$

$$
\left[\begin{array}{l}
E_{1}^{+} \\
E_{1}^{-}
\end{array}\right]=M\left[\begin{array}{l}
E_{n+1}^{+} \\
E_{n+1}^{-}
\end{array}\right],
$$

which is obtained by multiplying: the transition matrix $T$ through the interface, and the transmission matrix in a dielectric medium $P_{d}$ :

$$
M_{T M}=T \cdot P_{d}=\left[\begin{array}{cc}
1+\zeta_{T M} / 2 & \zeta_{T M} / 2 \\
\zeta_{T M} / 2 & 1-\zeta_{T M} / 2
\end{array}\right]\left[\begin{array}{cc}
e^{-i k_{z} d} & 0 \\
0 & e^{i k_{z} d}
\end{array}\right]
$$

Here $\zeta_{T M}=\sigma(\omega, \mu, \Gamma, T) k_{z} / \varepsilon \varepsilon_{0} \omega$ stands for the so-called wave impedance, $\sigma(\omega, \mu, \Gamma)$ denotes the conductivity of graphene, determined by the Kubo formula (1). Note that a wave is considered to be polarized parallel to the graphene sheet at the interface, since it is for this wave that the hyperbolic dependence of its dielectric properties is observed.

To find the dispersion relation, we consider a wave propagating in the direction of the $\mathrm{z}$ axis. This wave is weakened due to passing through each layer of the dielectric, so that in each layer of an infinite periodic structure the following conditions hold

$$
\begin{aligned}
& E_{n+1}^{+}=E_{n}^{+} e^{-\alpha d}, \\
& E_{n+1}^{-}=E_{n}^{-} e^{-\alpha d} .
\end{aligned}
$$

Solving equations (6) - (7) together with (4), it is not difficult to obtain the following homogeneous equation in the matrix form

$$
\left(\begin{array}{cc}
M_{11}-e^{\alpha d} & M_{12} \\
M_{21} & M_{22}-e^{\alpha d}
\end{array}\right)\left(\begin{array}{l}
E_{n+1}^{+} \\
E_{n+1}^{-}
\end{array}\right)=0
$$

whose nonzero solution only exists if the determinant of the matrix turns zero

$$
M_{11} M_{22}+e^{2 \alpha d}-\left(M_{11}+M_{22}\right) e^{\alpha d}-M_{12} M_{21}=0 .
$$

Here, $\alpha$ is the Bloch wave number, $d$ designates the thickness of the dielectric gap. 
Recalling that the condition $M_{11} M_{22}-M_{12} M_{21}=1$ must always be satisfied for a periodic structure, expression (9) is simplified to the following form:

$$
\operatorname{Cosh}(\alpha d)=\left(M_{11}+M_{22}\right) / 2 \text {. }
$$

If $\alpha$ is considered a complex number $\alpha=\beta+i \kappa, \quad$ at which $\operatorname{Cosh}(\alpha d)=\operatorname{Cosh}(\beta d) \operatorname{Cos}(\kappa d)+i \operatorname{Sinh}(\beta d) \operatorname{Sin}(\kappa d)$ , then, two limiting cases arise. Of interest is only the first case that corresponds to an undamped wave in the periodic structure and strictly determines its bandwidth

$$
\begin{gathered}
\operatorname{Cos}(\kappa d)=\frac{M_{11}+M_{22}}{2}= \\
=\operatorname{Cos}\left(k_{z} d\right)-i \frac{\zeta_{T M}}{2} \operatorname{Sin}\left(k_{z} d\right)
\end{gathered}
$$

The dispersion relation (10) describes the law of propagation of a TM polarized wave in a periodic photon structure. It should be noted that in the subwave regime, at which $k_{z} d \square 1$ and $\beta d \square 1$, expression (10) turns into the dispersion relation obtained on the basis of the effective medium model:

$$
\kappa^{2}=\varepsilon_{t} k_{0}^{2}-\frac{\varepsilon_{t}}{\varepsilon} k_{\perp}^{2},
$$

where $\varepsilon_{t}=\varepsilon-i \sigma(\omega, \mu, \Gamma, T) / \varepsilon_{0} \omega d$ denotes the perpendicular component of the permittivity.

Figure 3 shows the dependence of $\kappa=\kappa^{\prime}+i \kappa^{\prime \prime}$ on the transverse component of the wave number $k_{\perp}$, normalized to $k_{0}$. For numerical calculations quartz $\mathrm{SiO}_{2}$ with the permittivity of $\varepsilon=4.2$ and the width of $80 \mathrm{~nm}$ has been chosen as a dielectric medium. It is clearly seen from the figure that for the small value of the dimensionless chemical potential $\mu=0.1$, the dependence $\kappa\left(k_{\perp}\right)$ is purely elliptic, see Figure $3 \mathrm{a}$, and the transition point of the curve corresponds to the value $\sqrt{\varepsilon}$. At $\mu=0.6$ the elliptic character of the curve goes over to the hyperbolic, see Figure 3b. Note that, in practice, an increase in the chemical potential is merely achieved by doping of the graphene or by applying an external electric field.
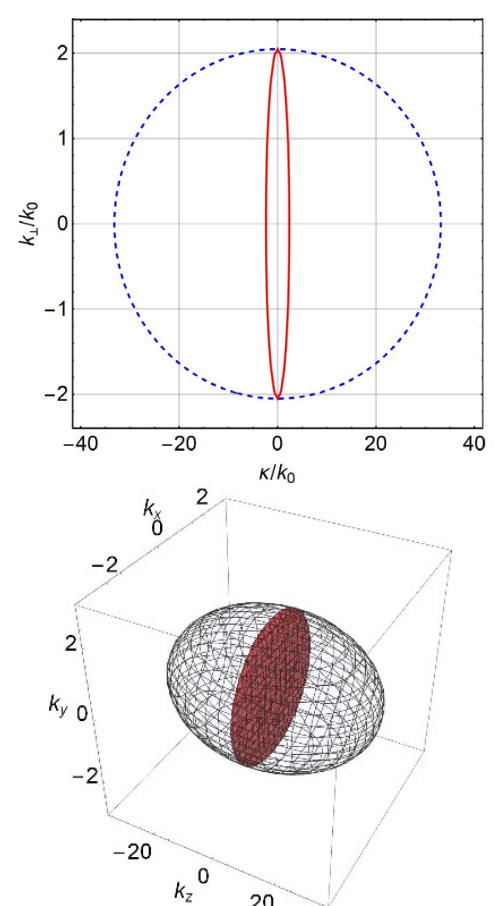

$\mu=0.1 \mathrm{eV}, T=300 \mathrm{~K}, \hbar \omega=0.91 \mathrm{eV}, \Gamma=0.043 \mathrm{eV}$

a) Elliptic behavior of the dispersion
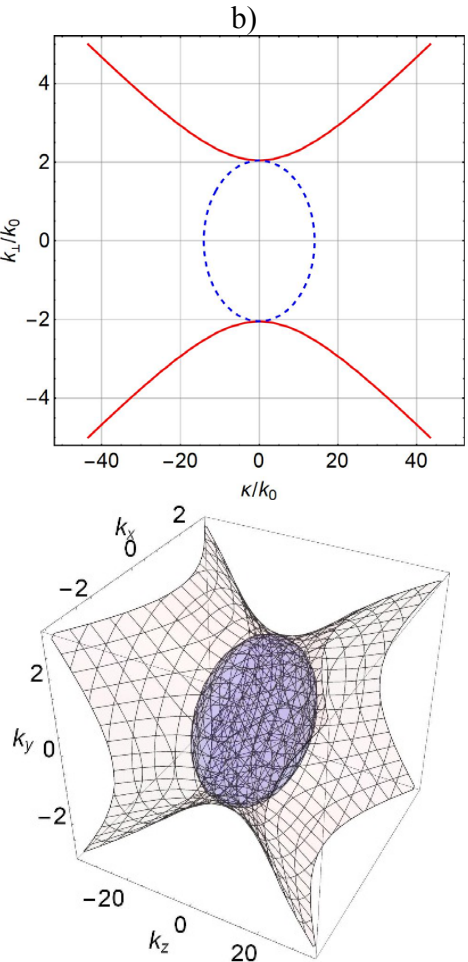

$\mu=0.6 \mathrm{eV}, T=300 \mathrm{~K}, \hbar \omega=0.91 \mathrm{eV}, \Gamma=0.043 \mathrm{eV}$

b) Hyperbolic behavior of the dispersion

Figure 3 -Dependence of $\kappa$ on $k_{\perp}=\sqrt{k_{x}^{2}+k_{y}^{2}}$ at different values of the chemical potential 


\section{Conclusion}

It is inferred from the figures above that when an external electric field, applied to graphene, is rather small such that the chemical potential $\mu$ is also not large, the dispersion law has purely elliptic shape. At the value of $\mu=0.6$, a transition of the dispersion law is revealed, which manifests itself in the appearance of forbidden bands for a planepolarized TM wave. In the frequency range, corresponding to the forbidden bands, the wave is completely reflected from the layered structure, whereas the transmitted wave has a minimum intensity. Based on the results obtained, it can be concluded that the graphene, due to its unique optical properties, is a more promising material for creating hyperbolic metamaterials than conventional metals. This statement is based on the fact that it is possible to control the light transmission through a layered structure by changing the chemical potential $\mu$.

\section{Reference}

1 P. Shekhar., J. Atkinson., Z. Jacob. Hyperbolic metamaterials: fundamentals and applications //Nano Convergence. - 2014. - Vol. 1. - P. 14.

2 V.A. Podolskiy., Alekseyev L., E.E. Narimanov. Strongly anisotropic media: the $\mathrm{THz}$ perspectives of left-handed materials // Journal of Modern Optics. - 2005. - Vol. 52. - P. 2343-2349.

3 Z. Jacob., L.V. Alekseyev., E. Narimanov. Optical hyperlens: Far-field imaging beyond the diffraction limit // Optics Express. - 2006. - Vol. 14. - P. 8247-8256.

4 Z. Liu., S. Durant., H. Lee., Y. Pikus., N. Fang., Y. Xiong., C. Sun., X. Zhang. Far-field optical superlens // Nano letters. - 2007. - Vol. 7. P. 403-408.
5 Z. Liu., H. Lee., Y. Xiong., C. Sun., X. Zhang. Far-field optical hyperlens magnifying subdiffraction-limited objects // Science. - 2007. - Vol. 315. - P. 1686-1686.

6 I.I. Smolyaninov., Y.J. Hung., C.C. Davis. Magnifying superlens in the visible frequency range // Science. - 2007. - Vol. 315. - P. 1699-01701.

7 J. Yao., X. Yang., X. Yin., G. Bartal., X. Zhang. Three-dimensional nanometer-scale optical cavities of indefinite medium // Proceedings of the National Academy of Sciences. - 2011. - Vol. 108. - P. 11327-11331.

8 V.A. Podolskiy., E.E. Narimanov. Strongly anisotropic waveguide as a nonmagnetic left-handed system // Physical Review B. - 2005. - Vol. 71. - P. 201101.

9 A.V. Kabashin., P. Evans., S. Pastkovsky., W. Hendren., G.A. Wurtz., R. Atkinson., R. Pollard., V.A. Podolskiy., A.V. Zayats. Plasmonic nanorod metamaterials for biosensing // Nature materials. 2009. - Vol. 8. - P. 867-871.

10 G.A. Wurtz., R. Pollard., W. Hendren., G.P. Wiederrecht., D.J. Gosztola., V.A. Podolskiy., A.V. Zayats. Designed ultrafast optical nonlinearity in a plasmonic nanorod metamaterial enhanced by nonlocality // Nature nanotechnology. - 2011. Vol. 6. - P. 107-111.

11 A. K. Geim., K. S. Novoselov. The rise of graphene // Nature materials. - 2007. - Vol. 6. - P. 183-191.

12 L. Ju., B. Geng., J. Horng., C. Girit., M. Martin., Z. Hao., H. A. Bechtel. Graphene plasmonics for tunable terahertz metamaterials // Nature nanotechnology - 2011. - Vol. 6. - P. 630634.

13. B. Francesco., Z. Sun. T., Hasan., A. C. Ferrari. Graphene photonics and optoelectronics // Nature photonics. - 2010. - Vol. 4. - P. 611-622.

14. K. S. A. Novoselov. et al. Two-dimensional gas of massless Dirac fermions in graphene // Nature. - 2005. - Vol. 438. - P.197-200. 